\title{
An Algorithm to Vehicle Scheduling Problem of AirPort Pickup and Delivery Service
}

\author{
Zhengzheng $\mathrm{Xu}^{1,2^{*}}$, Jiafu Tang ${ }^{2}$ \\ ${ }^{1}$ School of Business Administration, Northeastern University, Shenyang 110819, China; ${ }^{2}$ State Key Laboratory of Integrated Auto- \\ mation for Process Industries, Northeastern University, Shenyang 110819, China. \\ Email: *xuzz99@sina.com
}

Received July, 2013

\begin{abstract}
This paper studies the vehicle scheduling problem in cities for the pickup and delivery service. Considering customer point as vehicle collaboration point, we propose a two-stage algorithm based on customer point collaboration through the theory of optimization. In addition to considering the problem whether the isolated customer point is collaborative, the algorithm also takes into account the problem whether the customers on the vehicle (picking up multiple customer points) can transfer its customers to the other basic vehicle. Moreover, the selection of the type of basic vehicles is no longer single; we can choose different types of vehicles suitable for different vehicle capacities according to the total number of people to carry. Finally, we perform simulation analysis and simulation results show that the algorithm proposed in this paper is feasible and effective.
\end{abstract}

Keywords: Pickup and Delivery Service; Vehicle Collaboration; Satisfaction Degree; Isolated Customer Point

\section{Introduction}

The airport pickup and delivery service is a value-added service for the air ticket corporation to attract customers and enhance market competitiveness. The vehicle scheduling in the airport pickup and delivery service is significantly important for the modern logistics engineering and transportation. And it has received the extensive attention and studied well. The vehicle routing and scheduling problem of pickup and delivery of customers to airport belongs to the vehicle routing problem [1]. Some vehicles and trip number are unnecessary for the whole pickup and delivery service [2]. The vehicle scheduling problem is similar to the routing problem in communication networks where we are to find the optimal route according to the origin-destination flows [3, 4]. In the pickup and delivery service for customers, the vehicle scheduling via vehicle collaborations has received extensive attentions from academic and industrial circles.

Some vehicle scheduling methods have been proposed to this problem. Belfiore et al. studied the long-haul vehicle scheduling problems with working time rules [2]. Pop et al. used the integer programming to model the generalized vehicle routing problem and proposed two new models [1]. Wy et al. investigated the rollon-rolloff vehicle routing problem and brought forth a hybrid metaheuristic approach to overcome it [5]. Mester et al. studied both capacitated vehicle routing problem and vehicle routing problem with time window constraints, and then they presented an efficient evolution strategies algorithm to solve them [6]. The set-partitioning model was used to find the optimal vehicle routing and scheduling in the free pickup and delivery service in flight ticket sales companies [7]. Yan et al. studied the cash transportation vehicle routing and scheduling, and they developed a model to ensure cash conveyance safety and minimize the transportation cost [8].

This paper studies the vehicle scheduling problem in cities for the pickup and delivery service. We propose the concept of the isolated customer point through taking customer satisfaction and bypass limit as constraints. Considering customer point as vehicle collaboration point, we propose the vehicle collaboration rules about customer transfer. And a two-stage algorithm based on customer point collaboration is put forward through the theory of optimization. In addition to considering the problem whether the isolated customer point is collaborative, the algorithm also takes into account the problem whether the customers on the vehicle (picking up multiple customer points) can transfer its customers to the other basic vehicle. Moreover, the selection of the type of basic vehicles is no longer single; we can choose different types of vehicles suitable for different vehicle capacity according to the total number of people to carry. Finally, taking the airport pickup and delivery service of air 
ticketing company as a study case, we perform simulation analysis. Simulation results show that the algorithm proposed in this paper is feasible and effective.

\section{Problem Statement}

Consider as the research object the traveling passengers of a city to pickup and deliver in a plan period (one day). According to the basic traveling information of passengers (such as the traveling time and the pickup location of customers) in the plan period, air ticketing company is to arrange the number of vehicles dispatched, the departure time of each vehicle, customers to be picked up by each vehicle, pickup order for these customers, customer points to collaborate, selection of collaboration locations, and the pickup time. As a result, the company can achieve the high customer satisfaction degree and low operation cost. Basic vehicles are the vehicles that pick up customers from the depot to the airport. Collaborative vehicles refer to the vehicles that assist the basic vehicle to finish the pickup task from the depot but not arriving at the airport. Hence, for the characteristics of the problem itself, we give the following assumptions:

- In the pickup service, there is only one depot and one airport.

- Take the customers with the same pickup time in same location as the same customer point; the number of customers at each customer point is no more than the carrying capacity of each vehicle.

- Each vehicle serves one trip loop, and the whole process that the vehicle departs from the depot, arrives at the airport, and finally returns to the depot is regarded as one trip.

- Employ two kinds of vehicles, and collaborative vehicles select the vehicles with smaller vehicle capacity.

- The number of customers in basic vehicle is greater than or equal to the number of customers in its collaborative vehicle.

- During the whole pickup process, vehicles keep a constant speed and provide the service on time. Ignore the boarding time, and do not consider the delay and wait.

- Do not take the time required to transfer customers in the case of vehicle collaboration.

- Basic vehicles and collaborative vehicles will meet in the ideal state, namely without the waiting time.

Based on the above assumptions, this paper will study how to perform the collaboration between basic vehicles and collaborative vehicles at customer point during the pickup service of the single trip, in order to obtain the optimal routing and scheduling methods of the single trip and multiple vehicles.

In this section, the customers' satisfaction degree about the time arriving at the airport is used to measure whether customers are satisfied with the pickup service or not. And we can use the satisfaction degree function to describe the satisfaction degree of customers [8]. Considering the factor of vehicle collaboration and the satisfaction degree of customers at customer points, the satisfaction degree function of the customers at customer point $i$ based on the vehicles collaboration at customer points can be defined as:

$$
S\left(t_{i}\right)=\left\{\begin{array}{lc}
1-p_{i} & t_{i} \in\left[e_{i}, l_{i}\right] \\
\frac{e_{i}^{\prime}-t_{i}}{e_{i}^{\prime}-e_{i}}\left(1-p_{i}\right) & t_{i} \in\left[e_{i}^{\prime}, e_{i}\right] \\
\frac{l_{i}^{\prime}-t_{i}}{l_{i}^{\prime}-l_{i}}\left(1-p_{i}\right) & t_{i} \in\left[l_{i}, l_{i}^{\prime}\right] \\
0 & t_{i} \notin\left[e_{i}^{\prime}, l_{i}^{\prime}\right]
\end{array}\right.
$$

where $p_{i}$ is a constant and $0 \leq p_{i} \leq 1$; when the customers at customer point $i$ need not transfer, $p_{i}=0$, otherwise $p_{i}>0 ;\left[e_{i}, l_{i}\right]$ and $\left[e_{i}^{\prime}, l_{i}^{\prime}\right]$, respectively, indicate the soft time window [9] and hard time window [10] of reaching the airport, meeting $e_{i}^{\prime} \leq e_{i} \leq l_{i} \leq l_{i}^{\prime}$.

Assume that $U$ represents the duration of the work plan within a planning period (typically one day), $C=$ $\{1,2, \cdots, n\}$ indicates the customer point set, 0 represents the depot, $N=C \bigcup\{0\}$ denotes the set of customer points and the depot, -1 represents the airport, $N^{\prime}=C \bigcup\{0\} \bigcup\{-1\}$ denotes the set of all geographical locations during the customer pickup, $\omega_{i}$ represents the number of customers at customer point $i, \alpha$ is the level value of satisfaction degree that can be accepted by customers, $K_{B}=\{1,2, \cdots, n\}$ represents the basic vehicle set, $K_{C}=\{1,2, \cdots, m\}$ is the collaborative vehicle set, $Q_{C}$ denotes the passenger capacity of collaborative vehicles, $Q_{B}$ represents the maximum passenger capacity between the two kinds of basic vehicles, $T_{i j}$ is the travel time from customer point $i$ to customer point $j, d_{i j}$ represents the path distance form customer point $i$ to customer point $j, \quad \delta_{i k}$ denotes the arrival time of vehicle $k$ to customer point $i, \beta$ represents the bypass coefficient of customer pickup. Additionally, we assume that $z_{k}, y_{i k}, x_{i j k}$, and $v_{i j k}$ are the $0-1$ variables and they are the decision variables of vehicle $k$. When vehicle $k$ is used, $z_{k}=1$; when the customers at customer point $i$ is picked up by vehicle $k, y_{i k}=1$; when basic vehicle $k$ arrives form customer points $i$ to $j, x_{i j k}=1$; when collaborative vehicle $k$ arrives form customer points $i$ to $j, v_{i j k}=1$. In the collaboration process, the following constraints of collaboration are considered:

$$
\begin{array}{ll}
S\left(t_{i}\right) \geq \alpha, & \forall i \in C \\
\sum_{i \in C} \omega_{i} y_{i k} \leq Q_{B}, \quad & \forall k \in K_{B} \\
\sum_{i \in C} \omega_{i} y_{i k} \leq Q_{C}, \quad \forall k \in K_{c}
\end{array}
$$




$$
\sum_{k \in K_{B} \cup K_{C}}\left(t_{i}-\delta_{i k}\right) y_{i k} \leq \beta T_{i(-1)}, \forall i \in C
$$

Equation (2) denotes the constraints of customers' satisfaction degree. Equation (3) describes the constraints of the basic vehicles' capacity. Equation (4) denotes the constraints of the collaborative vehicles' capacity. Equation (5) is the limit of bypass time.

This paper designs a two-stage heuristic algorithm based on customer point collaboration. Customer point collaboration refers to the collaborative method that the collaborative vehicle transfers customers in it to the basic vehicle at a certain customer point. The first stage exploits the time sorting-based heuristic algorithm of prior clustering to generate the initial vehicle trips and access order of the customer points in each trip. The algorithm steps are as follows:

Step 1: Give the information of customer points, the lower limit $\alpha$ of satisfaction degree, and bypass limit coefficient $\beta$. Calculate the time window of arriving at airport that meets the lower limitation of satisfaction degree according to Equations (1) and (2).

Step 2: Sort the customer points in order according to the lower limit of the time window of arriving at airport. And form the sequence $K$.

Step 3: If $K$ is empty, stop and output the results.

Step 4: Select the first customer point in $K$ as the clustering point. Generate the set $S_{i}$ according to the sorting sequence of other customer points and the capacity limit of basic vehicles shown in Equation (3). Here the capacity of basic vehicle is limited to $Q_{B}=x$.

Step 5: if $S_{i}$ is not empty, we conduct full array of the customer pints in $S_{i}$, select the route with the shortest driving distance and denote it as $r_{i}$, and examine whether customer point $c_{j} \in r_{i} \quad\left(j=1,2, \ldots,\left|r_{i}\right|\right)$ meets the bypass limitation in Equation (5). If $c_{j}$ meets the constraint, then keep it in $r_{i}$. Or otherwise remove $c_{j}$ from $S_{i}$ and $r_{i}$ and put it into the set $E$. After finishing the whole process, put $r_{i}$ into the route set $R$. When $\left|S_{i}\right|=1$, put this customer point into the set E, delete $S_{i}$, and delete this customer point from $\mathrm{K}$. This is because the customer point is considered as the isolated customer point and is put into $E$ if there is only one customer point in the route.

Step 6: Delete from $K$ the customer points that have formed the cluster.

Step 7: Put the customer points in $\mathrm{E}$ in the time order according to the lower limit of time window of arriving at airport and form the sequence $L$. According to the constraints in Equations (2)-(5), select the customer point $e_{j} \in E$ to insert the route in $R$. Then form the new route, update the route set $R$, delete the customer points $e_{j}$ from $E$ and $L$, and go back to Step3.

Step 8: Conduct the second clustering for the customer points in E. Namely, repeat the above Steps 2-7 for the customer points in $E$. If the customer points in $E$ can form the new routes, then put these new routes into set $R$ and delete the corresponding customer points from $E$ and L.

At the first stage, we get the basic path set $R$ and isolated point set E. At the second stage, we mainly consider the customer satisfaction degree, vehicle capacity limitation, and bypass constraint based on the first stage. Using the selection rules of collaborative points, rearrange the route for the isolated points in a collaborative way. Then we take into account the possible collaboration between the collaborative vehicle picking up the customers of multiple customer points and the basic vehicle, and make the appropriate scheduling. The heuristic algorithm steps at the second phase are as follows:

Step 1: According to the isolated points in set $E$, consider the change of customers' satisfaction degree due to the vehicle collaboration. And recalculate the time window of arrive at the airport.

Step 2: If $L$ is empty, then remove all the basic path in path set $R$ collaborated by the isolated point to set $R 2$, output the result, and exit.

Step 3: Take out the isolated point from $\mathrm{L}$ in order, insert the basic path that has the same time window with this isolated point and meet the vehicle capacity constraints. According to the above Rules 1-3, select the appropriate the collaborative point, build the corresponding collaborative path, write down the basic path collaborated with isolated point, mark the collaborative customer point, remove this isolated point from set $E$ to collaborative path set $R_{1}$, update the arrival time window and the total number of the basic path which has been collaborated in set $R$. If not meeting the Rules 1-3, the isolated point does not participate in collaboration, separately construct the path, and place the built path into the path set $R$.

Step 4: Delete isolated points which have collaborated with basic path from $L$ and $E$, and then go back to Step 2 .

Step 5: Reorder the path in $R$ according to the lower limit of the arrival time window.

Step 6: Take the path $R_{i}$ form $R$. If $R_{i}$ is empty, output the result and exit.

Step 7: Calculate $w_{i}^{a} \pm f$ where $w_{i}^{a}$ denotes arrival time window of the path $R_{i}$ and $f$ represents penalty factor. Build the intersection set $U_{i}$ of $w_{i}^{a} \pm f$ and the time window of the existing path in $R 2$. If $U_{i}$ is not empty and the vehicle capacity constraint is satisfied, go to Step 4, or otherwise go back to Step 6 .

Step 8: Select the customer point $r$ in $R 2$ as the collaborative point. According to Rules 1-3, choose the collaborative point $C_{i}$ which make the collaborative mileage shortest and all customer points in $R_{i}$ meet the bypass restrictions. if $C_{i}$ is found, construct the collaborative path, write down the basic path and collaborative 
customer points collaborated by $R_{i}$, and remove $r$ from $R$ to the collaborative path set $R 3$, update the arrival time window and the total customer number of the basic path collaborated in $R 2$. If $C_{i}$ is not found, the path $R_{i}$ remains in $R$, and go back to Step 6 .

Step 9: Take $R_{i}$ from $R$. If $R_{i}$ is empty, remove all the basic paths in $R$ collaborated by the other basic paths into the path set $R 5$, output the result and exit.

Step 10: If $R_{i}$ has been collaborated, go to Step 9.

Step 11: Calculate $w_{i}^{a} \pm f$ where $w_{i}^{a}$ denotes the arrival time window of the path $R_{i}$ and $f$ represents the penalty factor. Build the intersection set $\bar{U}_{i}$ of $w_{i}^{a} \pm f$ and the time window of the basic paths in $R$ except $R_{i}$. If $\bar{U}_{i}$ is empty or the vehicle capacity constraint is not satisfied, go back to Step 9.

Step 12: Select the customer point $z$ from the basic paths in $R$ except $R_{i}$ in order as the collaborative point. By Rules 1-3, choose the collaborative point $Z_{i}$ which make the collaborative mileage shortest and all customer points in $R_{i}$ meet the bypass restrictions. If $Z_{i}$ is found, construct the collaborative path, write down the basic path and collaborative customer points collaborated by $R_{i}$, and remove $z$ from $R$ to the collaborative path set $R 4$, update the arrival time window and the total customer number of the basic path collaborated in $R$. If $Z_{i}$ is not found, the path $R_{i}$ remains in $R$, and go back to Step 9.

In summary, through the three parts at the second phase, we finally get the set $R=R \bigcup R$ of the basic paths not participating collaboration, collaborative set $C=R 1 \bigcup R 3 \bigcup R 4$ collaborating with the basic paths, the collaborated basic path set $D=R 2 \bigcup R 5$. Consequently, according to the number of customers on each path of $R$ and $D$, we can determine how to choose the kinds of basic vehicles. According to the information in $C$, we can determine the collaborative path and collaborative customer points. Thus the optimal adjustment is performed for the vehicle scheduling result formed at the first stage. The vehicle routing and scheduling problem of the single trip and multiple vehicles with the hybrid vehicle kinds is solved.

\section{Simulation Results and Analysis}

Here we use the pickup and delivery service of the airport as a study case to validate our algorithm. Assume there are 30 customer points to pick up for the flights of a period of 8:00 - 20:00, these customer points are distributed in the $55 \mathrm{~km} \times 55 \mathrm{~km}$ rectangular area, soft time window width is 20 minutes, hard time window width is 40 minutes, maximum capacity of basic vehicles is $Q_{B}=8$, maximum capacity of collaborative vehicles is $Q_{C}=4$, depot coordinate is $(35,37)$, airport coordinate is $(50,50)$, vehicle speed is $60 \mathrm{~km} / \mathrm{h}$, lower limit of customers' satisfaction degree is $\alpha=0.8$, bypass restriction coefficient is $\beta=1.5$ and $p_{i}=0.1$.

The corresponding information of 30 customer points is shown in Table 1. To facilitate the statement, we give the abbreviations in the follow tables as follows: CP: Customer Point; VCP: Vehicle Coordination Point; LCP: Location of Customer Point; LHW: Lower bound of Hard time Windows; LSW: Lower bound of Soft time Windows; USW: Upper bound of Soft time Windows; UHW: Upper bound of Hard time Windows; NC: Number of Customers; VN: Vehicle Number; COP: Collaboration Point. Using the first-phase algorithm without the vehicle collaboration, we generate the initial trip and the access sequence of customer points in each trip. Then the minimum running mileage and average utilization ratio of vehicle capacity are attained when not considering the vehicle collaboration. Average utilization ratio of vehicle capacity can be denoted as:

Table 1. Information of customer points.

\begin{tabular}{ccccccccccc}
\hline CP & 1 & 2 & 3 & 4 & 5 & 6 & 7 & 8 & 9 & 10 \\
\hline LCP & $(42,34)$ & $(43,45)$ & $(30,25)$ & $(35,44)$ & $(39,36)$ & $(31,50)$ & $(34,30)$ & $(44,16)$ & $(29,51)$ & $(45,52)$ \\
LHW & $7: 25$ & $7: 35$ & $8: 50$ & $7: 20$ & $7: 30$ & $9: 05$ & $8: 30$ & $8: 45$ & $8: 50$ & $8: 55$ \\
LSW & $7: 35$ & $7: 45$ & $9: 00$ & $7: 30$ & $7: 40$ & $9: 15$ & $8: 40$ & $8: 55$ & $9: 00$ & $9: 05$ \\
USW & $7: 55$ & $8: 05$ & $9: 20$ & $7: 50$ & $8: 00$ & $9: 35$ & $9: 00$ & $9: 15$ & $9: 20$ & $9: 25$ \\
UHW & $8: 05$ & $8: 15$ & $9: 30$ & $8: 00$ & $8: 10$ & $9: 45$ & $9: 10$ & $9: 25$ & $9: 30$ & $9: 35$ \\
NC & 2 & 1 & 1 & 1 & 2 & 1 & 3 & 1 & 1 & 1 \\
CP & 11 & 12 & 13 & 14 & 15 & 16 & 17 & 18 & 19 & 20 \\
LCP & $(27,33)$ & $(32,21)$ & $(42,40)$ & $(35,40)$ & $(14,10)$ & $(47,35)$ & $(35,40)$ & $(38,30)$ & $(26,30)$ & $(46,38)$ \\
LHW & $11: 40$ & $18: 00$ & $17: 25$ & $10: 25$ & $16: 15$ & $17: 00$ & $17: 15$ & $15: 40$ & $12: 25$ & $11: 05$ \\
LSW & $11: 50$ & $18: 10$ & $17: 35$ & $10: 35$ & $16: 25$ & $17: 10$ & $17: 25$ & $15: 50$ & $12: 35$ & $11: 15$ \\
USW & $12: 10$ & $18: 30$ & $17: 55$ & $10: 55$ & $16: 45$ & $17: 30$ & $17: 45$ & $16: 10$ & $12: 55$ & $11: 35$ \\
UHW & $12: 20$ & $18: 40$ & $18: 05$ & $11: 05$ & $16: 55$ & $17: 40$ & $17: 55$ & $16: 20$ & $13: 05$ & $11: 45$ \\
NC & 2 & 1 & 1 & 2 & 1 & 1 & 1 & 2 & 2 & 2 \\
CP & 21 & 22 & 23 & 24 & 25 & 26 & 27 & 28 & 29 \\
LCP & $(39,27)$ & $(28,40)$ & $(32,32)$ & $(43,43)$ & $(29,44)$ & $(40,30)$ & $(47,37)$ & $(55,25)$ & $(39,29)$ & $(30,36)$ \\
LHW & $17: 25$ & $10: 25$ & $12: 00$ & $12: 25$ & $11: 05$ & $13: 25$ & $13: 35$ & $13: 30$ & $13: 30$ & $18: 15$ \\
LSW & $17: 35$ & $10: 35$ & $12: 10$ & $12: 35$ & $11: 15$ & $13: 35$ & $13: 45$ & $13: 40$ & $13: 40$ & $18: 25$ \\
USW & $17: 55$ & $10: 55$ & $12: 30$ & $12: 55$ & $11: 35$ & $13: 55$ & $14: 05$ & $14: 00$ & $14: 00$ & $18: 45$ \\
UHW & $18: 05$ & $11: 05$ & $12: 40$ & $13: 05$ & $11: 45$ & $14: 05$ & $14: 15$ & $14: 10$ & $14: 10$ & $18: 55$ \\
NC & 1 & 1 & 1 & 1 & 1 & 1 & 4 & 1 & 2 \\
\hline
\end{tabular}




$$
\bar{\alpha}=\frac{N_{b}+N_{c}}{n_{b} \times s_{b}+n_{c} \times s_{c}},
$$

where $N_{b}$ and $N_{c}$ denote the number of the total customers delivered by basic and collaborative vehicles, respectively, $n_{b}, s_{b}, n_{c}$, and $s_{c}$ represent the trip number and seat number of basic and collaborative vehicles, respectively. When not considering the collaboration, $N_{c}=0$ and $n_{c}=0$.

Simulation results show that without collaborations, the total running mileage of vehicles is: $1051.4 \mathrm{~km}$ and average utilization ratio of vehicle capacity $57.89 \%$. Based on the results at the first stage, we perform the heuristic algorithm at the second stage. By optimizing the results at the first stage, we can obtain the collaborative information of collaborative vehicles. Simulation results indicate that with collaborations, the total vehicle mileage is $1008.5 \mathrm{~km}$ and average utilization ratio of vehicle capacity is $61.90 \%$.

Here we, respectively, use the study cases with 10, 30, 50, 100, 150, 200, 400, 600, 800 and 1000 customer points to validate further the performance of our algorithm. The depot coordinate is at point $(35,37)$, while airport coordinate is at point $(50,50)$. According to the distribution of the customer point locations, we discuss the two cases, namely customer points mainly distributed near the depot or airport. And the locations of all the customer points are randomly distributed in the circle whose center is the depot or airport with radius by $20 \mathrm{~km}$.

For collaborations and non-collaborations, we obtain the vehicle total mileage, the average utilization ratio of basic vehicles, and average satisfaction degree of customers for the different study case. From Figures 1-4, we can see that in contrast to collaborations, average satisfaction degree of customers decrease a little after collaborating. However, Figures 1-4 also tell us that the

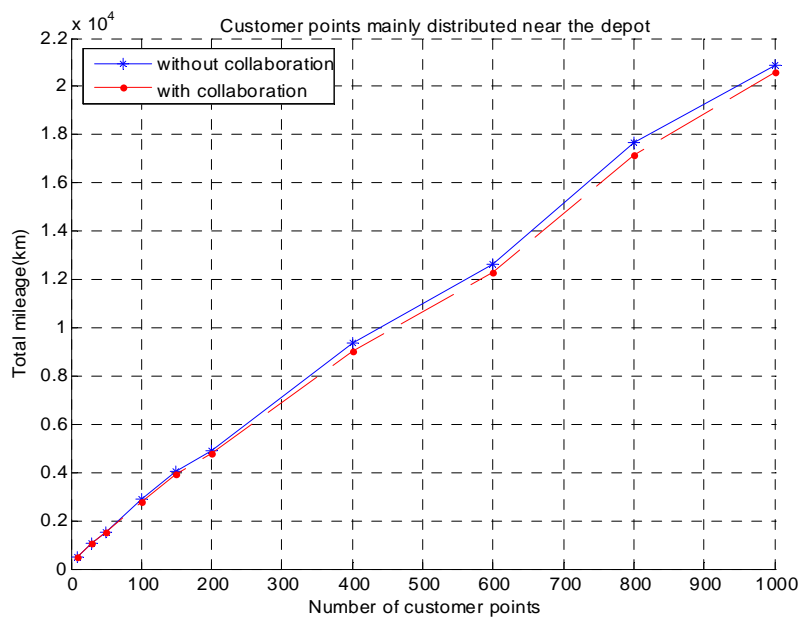

Figure 1. Total mileage with customer points mainly near the depot.

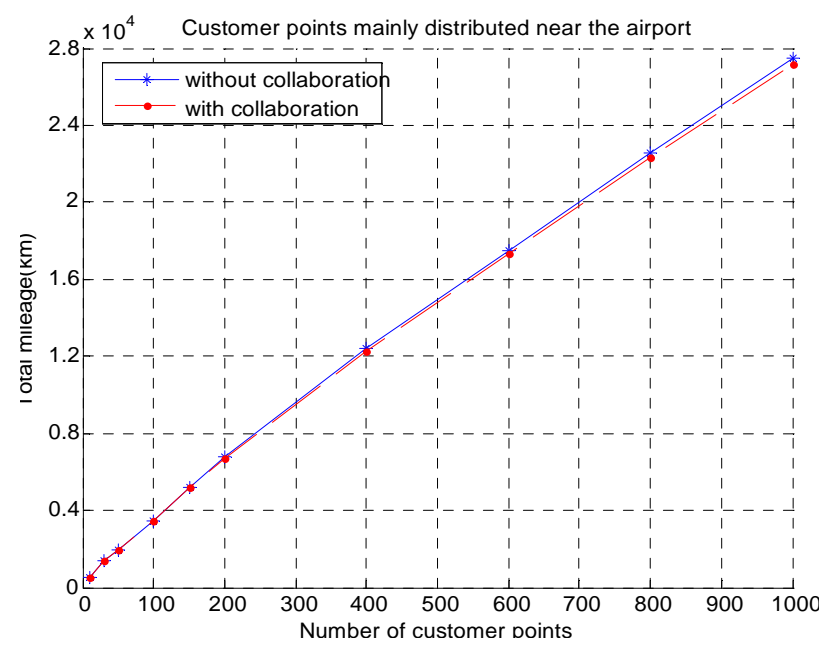

Figure 2. Total mileage with customer points mainly near the airport.

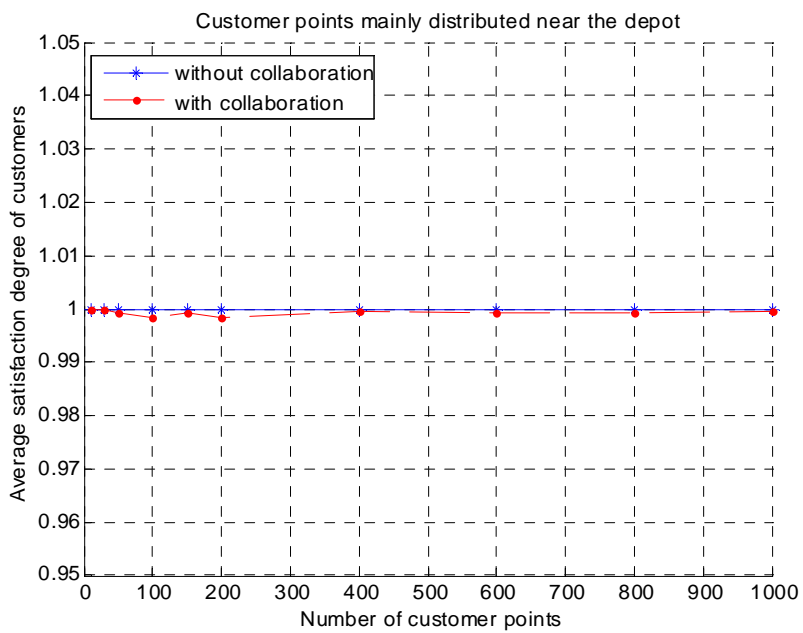

Figure 3. Average satisfaction degree of customers with customer points mainly near the depot.

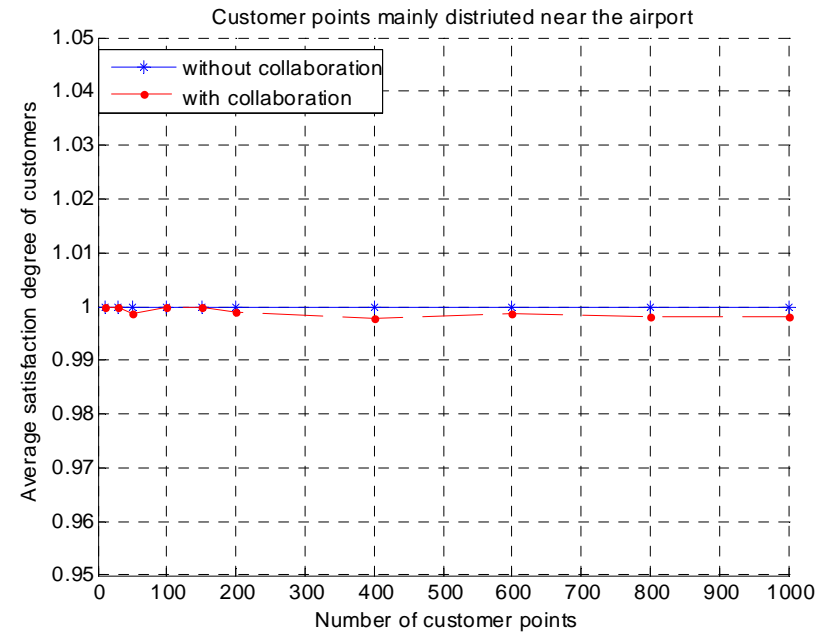

Figure 4. Average satisfaction degree of customers with customer points mainly near the airport. 
total mileage with collaborations is much less than that without collaborations. And we also find that the reduced total mileage is up to $4 \%$. This is what we expect. By the vehicle collaborations, the satisfaction degree of customers in the collaborative vehicles is to decrease. As a result, this is to affect the average satisfaction degree of customers. The reduced mileage is mainly because the collaborative vehicles need not go to the airport. After using the heuristic algorithm at the second stage, although the average satisfaction degree of customers decreases a little, the total running mileage of vehicles is reduced. And thus the vehicle scheduling cost is also reduced. Hence, simulation results indicate that the algorithm proposed in this paper can be used for all the study cases. This tells us that the algorithm proposed in this paper is effective and feasible.

\section{Conclusions}

This paper studies the two vehicle kinds' collaborative problems of the pickup and delivery service. By introducing the vehicle collaborations, a two-stage heuristic algorithm is proposed. Considering the collaborations between isolated customer points and basic vehicles, we make the basic vehicle picking up the customers at the multiple customer points collaborating with other basic vehicles. And the customers at the different customer points are put into the same basic vehicle as possible and then are picked up to the airport. In such a case, the average utilization ratio of vehicle capacity is improved. Moreover, simulation results that in the case of keeping the appropriate customers' satisfaction degree; we can perform the better vehicle scheduling. As a result, the cost of the company can be significantly reduced. Future studies need to consider the actual situations, such as multiple trips, waiting time when transferring, global collaboration, and dynamic insertion customers.

\section{Acknowledgements}

This work was supported in part by the National Natural Science Foundation of China (Nos. 71021061,
61273204), and the Fundamental Research Funds for the Central Universities (No. N090204001). The authors wish to thank the reviewers for their helpful comments.

\section{REFERENCES}

[1] P. Pop, I. Kara and A. Marc, "New Mathematical Models of the Generalized Vehicle Routing Problem and Extensions,” Applied Mathematical Modelling, 2012, Vol. 36, No. 1, pp. 97-107.doi:10.1016/j.apm.2011.05.037

[2] P. Belfiore and H. Yoshizaki, "Heuristic Methods for the Fleet Size and Mix Vehicle Routing Problem with Time Windows and Split Deliveries," Computers and Industrial Engineering, Vol. 64, No. 2, 2013, pp. 589-601. doi:10.1016/j.cie.2012.11.007

[3] D. Jiang and G. Hu, "GARCH Model-based Large-scale IP Traffic Matrix Estimation,” IEEE Communications Letters, Vol. 13, No. 1, 2009, pp. 52-54. doi:10.1109/LCOMM.2008.081271

[4] D. Jiang and G. Hu, "An Accurate Approach to Large-scale IP Traffic Matrix Estimation,” IEICE Transactions on Communications, Vol. E92B, No. 1, 2009, pp. 322-325.

[5] J. Wy and B. Kim, "A Hybrid Metaheuristic Approach for the Rollon-rolloff Vehicle Routing Problem," Computers and Operations Research, Vol. 40, No. 8, 2013, pp. 1947-1952.doi:10.1016/j.cor.2013.03.006

[6] D. Mester, O. Braysy and W. Dullaert, “A Multi-parametric Evolution Strategies Algorithm for Vehicle Routing Problems,” Expert Systems with Applications, Vol. 32, No. 2, 2007, pp. 508-517. doi:10.1016/j.eswa.2005.12.014

[7] M. Rancourt, J. Cordeau and G. Laporte, "Long-Haul Vehicle Routing and Scheduling with Working Hour Rules,” Transportation Science, Vol. 47, No. 1, 2013, pp. 81-107.doi:10.1287/trsc.1120.0417

[8] S. Yan, S. Wang and M. Wu, "A Model with a Solution Algorithm for the Cash Transportation Vehicle Routing and Scheduling Proble," Computers and Industrial Engineering, Vol. 63, No. 2, 2012, pp. 464-473. doi:10.1016/j.cie.2012.04.004

[9] Z. Xu and J. Tang, "A Coordination-based Two-stage Algorithm for Pickup and Delivery Customer to Airport Service,” in Proc. of ICMSEM’13, 2013, pp. 1-9. 